\title{
The Generalized Difference Operator of the $\mathbf{n}^{\text {th }}$ Kind
}

\author{
M.Vijayalakshmi ${ }^{1}$, J.Manigandan ${ }^{2}$ and M.V.Suresh ${ }^{3}$ \\ ${ }^{1}$ Research scholar, Department of Mathematics, SPIHER, Avadi, Chennai, INDIA \\ ${ }^{2}$ Assistant Professor, Department of Mathematics, SPIHER, Avadi, Chennai, INDIA \\ ${ }^{3}$ Assistant Professor, Department of Mathematics, SPIHER, Avadi, Chennai, INDIA
}

${ }^{1}$ Corresponding Author: viji.smmani78@gmail.com

\begin{abstract}
In this chapter, the authors extend the theory of the generalized difference Operator $\Delta_{\mathbf{L}}$ to the generalized difference operator of the $n^{t h}$ kind denoted by $\Delta_{L}$ Where $L$ $=L=\left\{l_{1}, l_{2}, \ldots . . l_{n}\right\}$ of positive reals $l_{1}, l_{2}, \ldots . . l_{n}$ and obtain some interesting results on the relation between the generalized polynomial factorial of the first kind, $n^{\text {th }}$ kind and algebraic polynomials. Also formulae for the sum of the general partial sums of products of several powers of consecutive terms of an Arithmetic progression in number theory are derived.
\end{abstract}

Keywords-- Difference Operator, Difference Equations, Arithmetic Progressions

\section{INTRODUCTION}

The theory of difference equations is based on the operator $\Delta$ defined as

$$
\Delta \mathrm{x}(\mathrm{n})=\mathrm{x}(\mathrm{n}+1)-\mathrm{x}(\mathrm{n}), \mathrm{n} \in \mathrm{W}
$$

Where $\mathrm{W}=\{-3,-2,-1,0,1,2,3, \cdots$,$\} . Even$ though many authors $[1,9-11]$, have defined $\Delta$ as
(2) $\quad \Delta x(n)=x(n+\ell)-x(n), \ell \in N$, no significant progress took place on this line. But recently, when we took up the definition of $\Delta$ as given in (2) and developed the theory of difference equations in a different direction, we obtained some interesting results in number theory. For convenience, we labelled the operator $\Delta$ defined by (2) as $\Delta_{\ell}, \ell \in \mathrm{W}$, named it as the generalized difference operator and by defining its inverse $\Delta_{l}-1 \ell$ we obtained many interesting results in number theory.

The theory was then extended for real $\ell \in(0, \infty)$ and $\Delta_{-\ell} \mathrm{x}(\mathrm{n})=\mathrm{x}(\mathrm{n}-\ell)-\mathrm{x}(\mathrm{n})$ and again many useful results were obtained in number theory. By extending the study for sequences of complex numbers and $\ell$ to be real, some new qualitative properties like rotatory, expanding and shrinking, spiral and web like were studied for the solutions of difference equations involving $\Delta_{\ell}$.

The results obtained can be found in [3-8].With this background, in this paper, we develop theory for $\Delta_{\mathrm{L}}$, the generalized difference operator of the $n^{\text {th }}$ kind and obtain some significant results, relations and formulae in number theory using Stirling numbers of the second kind, $\mathrm{S}_{\mathrm{r}}{ }^{\mathrm{n}}$. Throughout this paper, we make use of the following assumptions.

(i) $\ell, \ell 1, \ell 2, \ldots, \ell \mathrm{n}$ are real numbers, $\mathrm{C}$ is the set of all complex numbers,

(ii) $\mathrm{cj}, \mathrm{c} 0 \mathrm{j}, \mathrm{c} 1 \mathrm{j}, \ldots, \mathrm{c}(\mathrm{n}-1) \mathrm{j}$ are constants, $[\mathrm{x}]=$ integer part of $\mathrm{x}$,

(iii) where $0 !=1, r !=1,2, \ldots r$,

(iv) $\mathrm{W}(\mathrm{a})=\{\mathrm{a}, \mathrm{a}+1, \mathrm{a}+2, \ldots\}, \mathrm{W} \ell(\mathrm{j})=\{\mathrm{j}, \mathrm{j}+\ell, \mathrm{j}+2 \ell, \ldots\}$,

(v) $\mathrm{L}=\{\ell 1, \ell 2, \ldots, \ell \mathrm{n}\}$,

(vi) $0(\mathrm{~L})=\{\varphi\}, \varphi$ denotes the empty set,

(vii) $1(\mathrm{~L})=\{\{\ell 1\},\{\ell 2\}, \ldots,\{\ell \mathrm{n}\}\}$,

(viii) $2(\mathrm{~L})=\{\{\ell 1, \ell 2\},\{\ell 1, \ell 3\}, \ldots,\{\ell 1, \ell \mathrm{n}\},\{\ell 2, \ell 3\}, \ldots,\{\ell 2, \ell \mathrm{n}\}, \ldots,\{\ell \mathrm{n}-1, \ell \mathrm{n}\}\}$,

(ix) $(\mathrm{n}-1)(\mathrm{L})=\{\{\ell 1, \ell 2, \ldots, \ell \mathrm{n}-1\},\{\ell 1, \ell 2, \ldots, \ell \mathrm{n}-2, \ell \mathrm{n}\}, \ldots,\{\ell 2, \ell 3, \ldots, \ell \mathrm{n}\}\}$,

(x) $n(L)=\{\{\ell 1, \ell 2, \ldots, \ell n\}\}$,

(xi) In general, $r(L)=$ the set of all subsets of size $r$ of the set $L$ and

(xii) $\wp(\mathrm{L})=\mathrm{nS} \mathrm{r}=0 \mathrm{r}(\mathrm{L})$, the power set of $\mathrm{L}$.

\section{PRELIMINARIES}

In this section, we present some basic definitions and preliminary results which will be useful for further discussion.
Definition-1: For a function $\mathrm{u}(\mathrm{k}), \mathrm{k} \in[0, \infty)$, the generalized difference operator $\Delta_{l}$ is defined by

$$
\Delta_{l} \mathrm{u}(\mathrm{k})=
$$

$\mathrm{u}(\mathrm{k}+\mathrm{l})-\mathrm{u}(\mathrm{k})$.

Definition-2: The generalized difference operator of the $\mathrm{n}^{\text {th }}$-kind, denoted as $\Delta_{\mathrm{L}}$ for the function $\mathrm{u}(\mathrm{k}), \mathrm{k} \in[0, \infty)$, is defined as 
Note that $\Delta_{\mathrm{L}}=\Delta_{l_{1}} \Delta_{l_{2}} \ldots \Delta_{l_{n}}$.

$$
\Delta_{\mathrm{L}} \mathrm{u}(\mathrm{k})=\sum_{\mathrm{r}=-\mathrm{n}}^{\mathrm{n}}(-1)^{\mathrm{n}-(-\mathrm{n})}\left\{\sum_{\mathrm{A} \in \mathrm{p}=\mathrm{Ur}(\mathrm{L})} \mathrm{u}\left(\mathrm{k}+\sum_{l \in \mathrm{A}} l\right)\right\} .
$$

$$
\text { where } k_{L}^{(t)}=k(k-l)(k-2 l)(k-3 l) \ldots(k-(t-1) l) \text {. }
$$

Definition-3: The generalized polynomial factorial of the $\mathrm{n}^{\text {th }}$-kind, defined as

$$
\mathrm{k}_{\mathrm{L}}^{(\mathrm{t})}=\sum_{\mathrm{r}=1}^{\mathrm{n}}(-1)^{\mathrm{n}-(-\mathrm{n})}\left\{\sum_{\mathrm{A} \in \mathrm{r}(\mathrm{L})(\mathrm{p}(\mathrm{l})}\left(\left(\mathrm{k}+\sum_{l \mathrm{i} \in \mathrm{A}-\left\{l_{\mathrm{i}}\right\}} l\right)_{l_{i}}^{(t)}\right)\right\},
$$

Definition-4: If $l \in(0, \infty)$ and $\mathrm{n} \in \mathrm{N}(1)$, then the inverse operator $\Delta_{l}^{-1}$

Is defined as if

$$
\Delta_{l}(z(k))=u(k), \text { then } z(k)=\Delta_{l}^{-1}(u(k))+c_{j},
$$

Where $\mathrm{c}_{\mathrm{j}}$ is a constant for all $k \in N_{l}(j), j=k-\left[\frac{k}{l}\right] l$.

The inverse of the gereralized difference operator of the $\mathrm{n}^{\text {th }}$ - kind denoted by $\Delta_{\mathrm{L}}^{-1}$ is defined as if $\Delta_{\mathrm{L}} \mathrm{z}(\mathrm{k})=\mathrm{u}(\mathrm{k})$, then

$$
\begin{gathered}
z(k)=\Delta_{L}^{-1} u(k)+c_{(n-1) j} \frac{k_{l_{n-1}}^{(n-1)}}{(n-1) ! l_{n-1}^{n-1}}+c_{(n-2) j} \frac{k_{l_{n-2}}^{(n-2)}}{(n-2) ! l_{n-2}^{n-2}} \\
+\cdots+c_{2_{j}} \frac{k_{l_{2}}^{(2)}}{(2) ! l_{2}^{2}}+c_{1_{j}} \frac{k}{l}+c_{0_{j}}, \\
\text { where } c_{\mathrm{ij}}^{\prime} \mathrm{s} \text { are constants. In general } \Delta_{\mathrm{L}}^{-\mathrm{m}}=\Delta_{\mathrm{L}}^{-1}\left(\Delta_{\mathrm{L}}^{-(\mathrm{m}-1)}\right) .
\end{gathered}
$$

Lemma 6. If the Stirling numbers of the first kind is given by

$$
\mathrm{s}_{\mathrm{n}}^{\mathrm{n}}=1, \mathrm{~s}_{\mathrm{r}}^{\mathrm{n}}=0 \text { for } \mathrm{r}<1 \text { and } r \geq(\mathrm{n}+1) \text {, ands } \mathrm{s}_{\mathrm{r}}^{\mathrm{n}+1}=\mathrm{s}_{\mathrm{r}-1}^{\mathrm{n}}-\mathrm{ns}_{\mathrm{r}}^{\mathrm{n}} \text { for } \mathrm{r} \geq 1 \text {, }
$$

Then

$$
\sum_{r=1}^{n}\left(s_{r}^{n} l^{n-r} k^{r}\right)=k_{l}^{(n)} .
$$

Lemma 5.2.7. [14] If $\mathrm{s}_{\mathrm{r}}^{\mathrm{n}} \mathrm{s}$ are the stirling numbers of the second kind, then

$$
k^{n}=\sum_{r=1}^{n} s_{r}^{n} l^{n-r} k_{l}^{(r)} .
$$

\section{MAIN RESULTS}

In this section, we present the formula for sum of general partial sums of products of consecutive terms of higher powers of an arithmetic progression.

\section{Theorem 1}

$$
\begin{gathered}
\text { If } n \in N(2), l \in(0, \infty) \text { and } k \in(n l, \infty) \text {, then } \\
\Delta_{l, l, l, \ldots, l}^{-1} u(k)=\sum_{\substack{r_{n}=2 \\
r_{n}^{*}}}^{r_{n-1}^{*}} \sum_{r_{n-1}=1}^{r_{n-2}^{*}} \ldots \sum_{r_{n-2}=1}^{r_{2}^{*}} \sum_{r_{2}=1}^{r_{1}^{*}} u\left(k-r_{n} l-r_{n-1} l-\cdots-r_{2} l-r_{1} l\right) \\
+c_{(n-1) j}\left(\frac{k_{l}^{(n-1)}}{(n-1) ! l^{n-1}}\right)+c_{(n-2) j}\left(\frac{k_{l}^{(n-2)}}{(n-2) ! l^{n-2}}\right)+\cdots+c_{1 j}\left(\frac{k_{l}^{(1)}}{l}\right)+c_{0 j},(8) \\
\text { where } r_{n}^{*}=\left[\frac{k}{l}\right] l, r_{n-i}^{*}=r_{n-(i-1)}^{*} \text { for } i=1,2, \ldots, n-1 \text { and } \\
\mathrm{c}_{0 \mathrm{j}}, \mathrm{c}_{1 \mathrm{j}}, \ldots, \mathrm{c}_{(\mathrm{n}-1) \mathrm{j}} \mathrm{s} \text { are constants for all } \mathrm{k} \in N_{l}(\mathrm{j}), \mathrm{j}=\mathrm{k}-\left[\frac{\mathrm{k}}{l}\right] l \text { and when } \mathrm{n}=1, \\
\Delta_{l}^{-1} u(k)=\sum_{r=1}^{\left[\frac{k}{l}\right]} u(k-r l)+c_{0 j} .
\end{gathered}
$$




\section{Proof:}

Definition 1, we can obtain

$$
\text { Since } \Delta_{l}\left\{\sum_{r=1}^{\left[\frac{k}{l}\right]} u(k-r l)\right\}=\sum_{r=1}^{\left[\frac{k}{l}\right]+1} u(k+l-r l)-\sum_{r=1}^{\left[\frac{k}{l}\right]} u(k-r l)=u(k),
$$

$$
\sum_{r=1}^{\left[\frac{k}{l}\right]} u(k-r l)=\Delta_{l}^{-1} u(k)+c_{0 j} .
$$

Since $\Delta_{l, l}^{-1}=\Delta_{l}^{-1}\left(\Delta_{l}^{-1}\right)$, by taking $\Delta_{l}^{-1}$ on bothsides of (9) and again applying (9), we get

$$
\sum_{r=1}^{\left[\frac{k}{l}\right]\left[\frac{k}{l}\right]-r_{2}} \sum_{r=1}^{[} u\left(k-r_{2} l-r_{1} l\right)+\frac{k}{l} c_{1 j}+c_{0 j}=\Delta_{l, l}^{-1} u(k) .
$$

proceeding in this way and using the relation $\Delta_{l, l, l, \ldots, l}=\Delta_{l} \Delta_{l} \ldots \Delta_{l}$, we get(8) and hence the proof of the theorem.

\section{Lemma 1}

\section{Proof:}

If $\mathrm{m}, \mathrm{n}$ are positive integers, $\mathrm{l}$ is a real and $\mathrm{m}>n l$, then

(i) $(k-(n-1) l)^{m}-(n-1)(k-(n-2) l)^{m}+(-1)^{n-1} k^{m}$

$$
=\frac{1}{n} \sum_{r=1}^{m} s_{r}^{m} l^{m-r} k_{l, l, \ldots, l}^{(r)} \text {. }
$$

$$
\begin{aligned}
& (i i) \Delta_{l, l, \ldots, l}^{-1} k_{l}^{(m)}=\frac{k_{l, l, \ldots, l}^{(m+2 n-1)}}{n(m+1)(m+2) \ldots(m+2 n-1) l^{2 n-1}}+c_{(n-1) j}\left(\frac{k_{l}^{(n-1)}}{(n-1) ! l^{n-1}}\right) \\
& +c_{(n-2) j}\left(\frac{k_{l}^{(n-2)}}{(n-2) ! l^{n-2}}\right)_{m}+\cdots+c_{2 j} \frac{k_{l_{2}}^{(2)}}{(2) ! l_{2}^{2}}+c_{1 j}\left(\frac{k_{l}^{(1)}}{l}\right)+c_{0 j} . \\
& \text { (iii) } k_{l}^{(m)}=\frac{1}{n} \sum_{r=1}^{m} s_{r}^{m} l^{m-r} \Delta_{l, l, \ldots, l}^{-1} k_{l, l, \ldots, l}^{(r)}
\end{aligned}
$$

\section{Theorem 2}

\section{Theorem 4}

The proof follows from definitions $2,3,5$ and the stirling numbers.

If $m, n$ are positive integers, $l$ is a real and $m>n l$, then

$$
\begin{aligned}
& \sum_{r_{n}=2}^{r_{n}^{*}} \sum_{r_{n-1}}^{r_{n-1}^{*}} \sum_{r_{n-2}=1}^{r_{n-2}^{*}} \ldots \sum_{r_{2}=1}^{r_{2}^{*}} \sum_{r_{1}=0}^{r_{1}^{*}}\left(k-r_{n} l-r_{n-1} l-\cdots-r_{2} l-r_{1} l\right)_{l}^{(m)} \\
& =\frac{k_{l, l, \ldots, l}^{(m+2 n-1)}}{n(m+1)(m+2) \ldots(m+2 n-1) l^{2 n-1}}+c_{(n-1) j}\left(\frac{k_{l}^{(n-1)}}{(n-1) ! l^{n-1}}\right) \\
& +c_{(n-2) j}\left(\frac{k_{l}^{(n-2)}}{(n-2) ! l^{n-2}}\right)+\cdots+c_{2_{j}} \frac{k_{l_{2}}^{(2)}}{(2) ! l_{2}^{2}}+c_{1 j}\left(\frac{k_{l}^{(1)}}{l}\right)+c_{0 j},
\end{aligned}
$$

where $c_{\mathrm{ij}}^{\prime} \mathrm{s}$ are obtained by solving $\mathrm{n}$ equations by putting $\mathrm{k}=(\mathrm{m}+\mathrm{a}) l+\mathrm{j}$ for $\mathrm{a}=\mathrm{n}-1, \mathrm{n}, \mathrm{n}+1, \ldots, 2 \mathrm{n}-2$

Proof. The proof follows by )(11) and Theorem 1

The following theorem gives the formula for sum of $(n-1)$ times partial sums (ie., partial sums of partial sums of ... partial sums of) for products of $\mathrm{p}_{\mathrm{i}}^{\text {th }},(\mathrm{i}=1,2, \ldots, \mathrm{n})$ powers of $\mathrm{m}$ consecutive terms $k^{p_{1}}(k-l)^{p_{2}}$ $\ldots(k-(m-1))^{p_{m}}$ of an arithmetic progression $k, k-l, k-2 l, \ldots, j$, where $j=k-\left[\frac{k}{l}\right] l$.

Lets $\mathrm{r}_{\mathrm{r}}^{\mathrm{t}}$ be the stirling numbers of the second kind, $\mathrm{p}_{1}, \mathrm{p}_{2}, \mathrm{p}_{3}, \ldots \mathrm{p}_{\mathrm{m}}$. are positive integers and $\mathrm{k} \in\left[\mathrm{p}_{\mathrm{m}} l+\mathrm{j}, \infty\right)$, where $\mathrm{p}_{\mathrm{m}}=\mathrm{p}_{1}+\mathrm{p}_{2}+\cdots+\mathrm{p}_{\mathrm{m}}$. Then, 


$$
\begin{aligned}
& \sum_{r_{n}=2}^{r_{n}^{*}} \sum_{r_{n-1}=1}^{r_{n}^{*}-1} \sum_{r_{n-2}=1}^{r_{n}^{*}-2} \ldots \sum_{r_{2}=1}^{r_{2}^{*}} \sum_{r_{1}=0}^{r_{1}^{*}}\left[\prod_{t=1}^{m}\left(k-r_{n} l-r_{n-1} l-\cdots-r_{2} l-(t-1) l\right)^{p_{t}}\right] \\
& \sum_{i_{1}=0}^{p_{1}} \sum_{i_{2}=0}^{p_{2}} \sum_{i_{3}=0}^{p_{3}} \ldots \sum_{i_{m-1}}^{p_{m}} \sum_{r=1}^{p_{m}-\sum i_{s}}\left(\begin{array}{c}
p_{2} \\
i_{2}
\end{array}\right)\left(\begin{array}{c}
p_{3} \\
i_{2}
\end{array}\right) \ldots .\left(\begin{array}{c}
p_{m} \\
i_{m-1}
\end{array}\right)(-1)^{i_{1}}(-2)^{i_{2}} \ldots \\
& \times(-(m-1))^{i_{m-1}} s_{r}^{p_{m}-\sum i_{s}}(l)^{p_{m}-(r+n)} \frac{k_{l}^{(r+n)}}{\prod_{i=1}^{n}(r+i)}+c_{(n-1) j}\left(\frac{k_{l}^{(n-1)}}{(n-1) ! l^{n-1}}\right) \\
& +c_{(n-2) j}\left(\frac{k_{l}^{(n-2)}}{(n-2) ! l^{n-2}}\right)+\cdots+c_{2_{j}} \frac{k_{l}^{(2)}}{(2) ! l^{2}}+c_{1 j}\left(\frac{k_{l}^{(1)}}{l}\right)+c_{0 j}, \\
& \text { where } \sum \mathrm{i}_{\mathrm{s}}=\mathrm{i}_{1}+\mathrm{i}_{2}+\cdots+\mathrm{i}_{\mathrm{m}-1} \text { and the constants } \mathrm{c}_{(\mathrm{n}-1) \mathrm{j}}, \mathrm{c}_{(\mathrm{n}-2) \mathrm{j}}, \ldots, \mathrm{c}_{0 \mathrm{j}} \text { are } \\
& \text { given by solving the } \mathrm{n} \text { equations obtained by putting } \mathrm{k}=\left(\mathrm{p}_{\mathrm{m}}+\mathrm{a}\right) l+\mathrm{j} \text { for } \\
& \mathrm{a}=\mathrm{n}-1, \mathrm{n}, \mathrm{n}+1, \ldots, 2 \mathrm{n}-2 \text { in }(13) \\
& k^{p_{1}}(k-l)^{p_{2}}(k-2 l)^{p_{3}} \ldots(k-(m-1) l)^{p_{m}}=\sum_{i_{1}=0}^{p_{2}} \sum_{i_{2}=0}^{p_{3}} \sum_{i_{3}=0}^{p_{4}} \ldots \sum_{i_{m-1}}^{p_{m}} \sum_{r=1}^{p_{m}-\sum i_{s}}\left(\begin{array}{c}
p_{2} \\
i_{2}
\end{array}\right)\left(\begin{array}{c}
p_{3} \\
i_{2}
\end{array}\right) \ldots .\left(\begin{array}{c}
p_{m} \\
i_{m-1}
\end{array}\right)(-1)^{i_{1}}(-2)^{i_{2}} \ldots \\
& (-(m-1))^{i_{m-1}} s_{r}^{p_{m}-\sum i_{s}}(l)^{p_{m}-r} k_{l}^{(r)} \\
& \sum_{r_{2}=2}^{\left[\frac{k}{l}\right]} \sum_{r_{1}=0}^{\left[\frac{k}{l}\right]-r_{2}}\left[\prod_{t=1}^{m}\left(k-r_{n} l-r_{n-1} l-\cdots-r_{2} l-(t-1) l\right)^{p_{t}}\right] \\
& =\sum_{i_{1}=0}^{p_{2}} \sum_{i_{2}=0}^{p_{3}} \sum_{i_{3}=0}^{p_{4}} \ldots \sum_{i_{m-1}}^{p_{m}} \sum_{r=1}^{p_{m}-\sum i_{s}}\left(\begin{array}{c}
p_{2} \\
i_{2}
\end{array}\right)\left(\begin{array}{c}
p_{3} \\
i_{2}
\end{array}\right) \ldots .\left(\begin{array}{c}
p_{m} \\
i_{m-1}
\end{array}\right)(-1)^{i_{1}}(-2)^{i_{2}} \ldots \\
& (-(m-1))^{i_{m-1}} s_{r}^{p_{m}-\sum i_{s}}(l)^{p_{m}-(r+2)} \times\left(\frac{k_{l}^{(r+2)}}{(r+1)(r+2)}\right)+\frac{c_{i j} k}{l}+c_{0 j}
\end{aligned}
$$

where $c_{0 \mathrm{j}}$ and $\mathrm{c}_{1 \mathrm{j}}$ are constants obtained by solving the two simultaneous equations obtained by substituting $\mathrm{k}=\left(\mathrm{p}_{\mathrm{m}}+\mathrm{a}\right) l+\mathrm{j}$ for $\mathrm{a}=1,2$ in (15).

Proof. The proof follows by $n=2$ in Theorem 4 .

The following corollary shows the formula for sum of partial sums for products of $\mathrm{p}_{\mathrm{i}}^{\text {th }}$ powers of $\mathrm{m}$ consecutive terms $k^{p_{1}}(k-l)^{p_{2}} \ldots(k-(m-1) l)^{p_{m}}$ of an arithmetic progression $k, k-l, k-2 l, \ldots, j$, where $j=k-\left[\frac{k}{l}\right] l$.

Corollary 6. If $\mathrm{p}_{\mathrm{m}}, \sum_{\mathrm{s}}, \mathrm{s}_{\mathrm{r}}^{\mathrm{n}}, \mathrm{k}$ and 1 are as in theorem 4 ,

$$
\begin{gathered}
\sum_{r_{3}=2}^{\left[\frac{k}{l}\right]\left[\begin{array}{c}
k \\
l
\end{array}\right]-r_{3}} \sum_{r_{2}=1}^{\left[\begin{array}{l}
k \\
l
\end{array}\right]-r_{3}-r_{2}} \sum_{r_{1}=0}^{p_{2}}\left[\prod_{t=1}^{m}\left(k-r_{n} l-r_{n-1} l-\cdots-r_{2} l-(t-1) l\right)^{p_{t}}\right]= \\
\sum_{i_{1}=0}^{p_{2}} \sum_{i_{2}=0}^{p_{3}} \sum_{i_{3}=0}^{p_{4}} \ldots \sum_{i_{m-1}}^{p_{m}} \sum_{r=1}^{p_{m-\sum i_{s}}}\left(\begin{array}{c}
p_{2} \\
i_{2}
\end{array}\right)\left(\begin{array}{c}
p_{3} \\
i_{2}
\end{array}\right) \ldots .\left(\begin{array}{c}
p_{m} \\
i_{m-1}
\end{array}\right)(-1)^{i_{1}}(-2)^{i_{2}} \ldots(-(m-1))^{i_{m-1}} \\
s_{r}^{p_{m}-\sum i_{s}}(l)^{p_{m}-(r+3)} \times\left(\frac{k_{l}^{(r+3)}}{(r+1)(r+2)(r+3)}\right)+\frac{c_{2_{j}} k_{l}^{(2)}}{2 ! l^{2}}+c_{0 j},(16)
\end{gathered}
$$


where $\mathrm{c}_{0 \mathrm{j}}, \mathrm{c}_{1 \mathrm{j}}$ and $\mathrm{c}_{2 \mathrm{j}}$ are constants obtained by solving the two simultaneous equations obtained by substituting $\mathrm{k}=\left(\mathrm{p}_{\mathrm{m}}+\mathrm{a}\right) l+\mathrm{j}$ for $\mathrm{a}=2,3,4$ in (16).

Proof. The proof follows by $n=3$ in Theorem 4 .

\section{APPLICATIONS}

In this section, we present some examples to illustrate the main results. The following example is an illustration of Corollary 5.

\section{Examples 1}

Formula for sum of partial sums of products of $1^{\text {st }}, 2^{\text {nd }}$ and $3^{\text {rd }}$ powers of three consecutiveterms $(k(k-$ $l 2 k-2 l 3 o f$ A.P. $k, k-l$,

$$
\begin{gathered}
k-2 l, \ldots, j, \text { where } j=k-\left[\frac{k}{l}\right] l \text { is given by } \\
\sum_{t=2}^{\left[\frac{k}{l}\right]\left[\frac{k}{l}\right]-t} \sum_{s=0}^{k}(k-t l-s l)(k-l-t l-s l)^{2}(k-2 l-t l-s l)^{3}= \\
\frac{1}{56 l^{2}}\left[k_{l}^{(8)}-(7 l+j)_{l}^{(8)}\right]+\frac{1}{6 l}\left[k_{l}^{(7)}-(7 l+j)_{l}^{(7)}\right]+\frac{1}{3}\left[k_{l}^{(6)}-(7 l+j)_{l}^{(6)}\right] \\
+\frac{l}{10}\left[k_{l}^{(5)}-(7 l+j)_{l}^{(5)}\right]+\sum_{t=2}^{7} \sum_{s=0}^{7-l}(7 l+j-t l-s l)(7 l+j-l-t l-s l)^{2} \\
(7 l+j-2 l-t l-s l)^{3} \\
\left(\frac{k-(7 l+j)}{l}\right)\left\{\sum_{s=1}^{5}(7 l+j-s l)(6 l+j-s l)^{2}(5 l+j-s l)^{3}-\frac{1}{56 l^{2}}\left[(8 l+j)_{l}^{(8)}-(7 l+j)_{l}^{(8)}\right]-\frac{1}{6 l}\left[(8 l+j)_{l}^{(7)}\right)\right. \\
\left.\left.-(7 l+j)_{l}^{(7)}\right]-\frac{1}{3}\left[(8 l+j)_{l}^{(6)}-(7 l+j)_{l}^{(6)}\right]+\frac{l}{10}\left[(8 l+j)_{l}^{(5)}-(7 l+j)_{l}^{(5)}\right]\right\}
\end{gathered}
$$

Solution: By taking $\mathrm{p}_{1}=1, \mathrm{p}_{2}=2, \mathrm{p}_{3}=3, \mathrm{~m}=3$ in corollary 5 , we find

$$
\begin{gathered}
\sum_{t=2}^{\left[\frac{k}{l}\right]} \sum_{s=0}^{\left[\frac{k}{l}\right]-t}(k-t l-s l)(k-l-t l-s l)^{2}(k-2 l-t l-s l)^{3}=\frac{k_{l}^{(8)}}{56 l^{2}}+\frac{k_{l}^{(7)}}{6 l} \\
+\frac{k_{l}^{(6)}}{3}+\frac{l k_{l}^{(5)}}{10}+c_{1_{j}} \frac{k}{l}+c_{0_{j}} .
\end{gathered}
$$

Putting $k=(7 l+j)$ and $k=(8 l+j)$ in $(17)$, we get

$$
\begin{aligned}
& \sum_{t=2}^{7} \sum_{s=0}^{7-t}(7 l+j-t l-s l)(7 l+j-t l-s l-l)^{2}(7 l+j-2 l-t l-s l)^{3}= \\
& \frac{(7 l+j)_{l}^{(8)}}{56 l^{2}}+\frac{(7 l+j)_{l}^{(7)}}{6 l}+\frac{(7 l+j)_{l}^{(6)}}{3}+\frac{l(7 l+j)_{l}^{(5)}}{10}+c_{1_{j}} \frac{(7 j+l)}{l}+c_{0_{j}} \cdot(18) \\
& \sum_{t=2}^{8} \sum_{s=0}^{8-t}(8 l+j-t l-s l)(8 l+j-t l-s l-l)^{2}(8 l+j-2 l-t l-s l)^{3}= \\
& \frac{(8 l+j)_{l}^{(8)}}{56 l^{2}}+\frac{(8 l+j)_{l}^{(7)}}{6 l}+\frac{(8 l+j)_{l}^{(6)}}{3}+\frac{l(8 l+j)_{l}^{(5)}}{10}+c_{1_{j}} \frac{(8 j+l)}{l}+c_{0_{j}} \cdot(19)
\end{aligned}
$$

Hence, $c_{0_{j}}$ and $c_{1_{j}}$ are obtained by solving (18)and (19). now the proof follows by substituting the values of $c_{0_{j}}$ and $c_{1_{j}}$ in (17).

In particular, taking $\mathrm{k}=38$ and $l=3$, we obtain

$$
\begin{gathered}
{\left[(32)(29)^{2}(26)^{3}+(29)(26)^{2}(23)^{3}+\cdots+(8)(5)^{2}(2)^{3}\right]+\left[(29)(26)^{2}(23)^{3}+\right.} \\
\left.(26)(23)^{2}(20)^{3}+\cdots+(8)(5)^{2}(2)^{3}\right]+\cdots+(8)(5)^{2}(2)^{3} \\
=\frac{1}{504}\left[(38)_{3}^{(8)}-(23)_{3}^{(8)}\right]+\frac{1}{18}\left[(38)_{3}^{(7)}-(23)_{3}^{(7)}\right]+\frac{1}{3}\left[(38)_{3}^{(6)}-(23)_{3}^{(6)}\right]+ \\
\frac{3}{10}\left[(38)_{3}^{(5)}-(23)_{3}^{(5)}\right]+\sum_{t=2}^{7} \sum_{s=0}^{7-t}(23-3 t-3 s)(20-3 t-3 s)^{2}(17-3 t-3 s)^{3}
\end{gathered}
$$




$$
\begin{gathered}
+(5)\left\{\sum_{s=1}^{5}\left[(23-3 s)(20-3 s)^{2}(17-3 s)^{3}\right]-\frac{1}{504}\left[(26)_{3}^{(8)}-(23)_{3}^{(8)}\right]-\frac{1}{18}\left[(26)_{3}^{(7)}-(23)_{3}^{(7)}\right]-\frac{1}{3}\left[(26)_{3}^{(6)}-(23)_{3}^{(6)}\right]\right. \\
\left.-\frac{3}{10}\left[(26)_{3}^{(5)}-(23)_{3}^{(5)}\right]\right\} \quad=1573644096
\end{gathered}
$$

The following example illustrates corollary 6 .

Example 2. Formula for sum of second partial sums of products of

$2^{\text {nd }}$ and $3^{\text {rd }}$ powers of two consecutive terms $\left(k^{2}(k-l)^{3}\right)$ of A.P. $k, k-l$,

$$
k-2 l, \ldots, j, \text { where } j=k-\left[\frac{k}{l}\right] l \text { is }
$$

$$
\begin{aligned}
& \sum_{t=2} \sum_{s=1} \sum_{r=0}(k-t l-s l-r l)^{2}(k-l-t l-s l-r l)^{3}= \\
& \frac{1}{336 l^{3}}\left[k_{l}^{(8)}-(7 l+j)_{l}^{(8)}\right]+\frac{1}{30 l^{2}}\left[k_{l}^{(7)}-(7 l+j)_{l}^{(7)}\right]+\frac{1}{12 l}\left[k_{l}^{(6)}-(7 l+j)_{l}^{(6)}\right] \\
& +\frac{l}{30}\left[k_{l}^{(5)}-(7 l+j)_{l}^{(5)}\right]+\sum_{t=2}^{7} \sum_{r=0}^{7-t}(7 l+j-t l-s l-r l)^{2}(7 l+j-2 l-t l-s l)^{3} \\
& \left(\frac{k-(7 l+j)}{l}\right)\left\{\sum_{t=2}^{8} \sum_{s=1}^{8-t}(8 l+j-t l-s l)^{2}(8 l+j-l-t l-s l)^{3}\right. \\
& \left.-\frac{1}{336 l^{3}}\left[(8 l+j)_{l}^{(8)}-(7 l+j)_{l}^{(8)}\right]-\frac{1}{30 l^{2}}\left[(8 l+j)_{l}^{(7)}\right)-(7 l+j)_{l}^{(7)}\right] \\
& -\frac{1}{12 l}\left[\left[(8 l+j)_{l}^{(6)}-(7 l+j)_{l}^{(6)}\right]+\frac{l}{30}\left[(8 l+j)_{l}^{(5)}-(7 l+j)_{l}^{(5)}\right]\right\} \\
& +\frac{1}{2 ! l^{2}}\left[k_{l}^{(2)}-(7 l+j)_{l}^{(2)}\right]+\left(\frac{k-(7 l+j)}{l}\right)\left[(8 l+j)_{l}^{(2)}-(7 l+j)_{l}^{(2)}\right] \\
& \left\{\sum_{s=1}^{5}(8 l+j-t l)^{2}(8 l+j-t l-l)^{3}-\frac{1}{336 l^{3}}\left[(9 l+j)_{l}^{(8)}-(7 l+j)_{l}^{(8)}\right]-\frac{1}{30 l^{2}}\left[(9 l+j)_{l}^{(7)}\right)-(7 l+j)_{l}^{(7)}\right]-\frac{1}{12 l}[(9 l \\
& \left.+j)_{l}^{(6)}-(7 l+j)_{l}^{(6)}\right]-\frac{1}{30}\left[(9 l+j)_{l}^{(5)}-(7 l+j)_{l}^{(5)}\right]-2\left(-\frac{1}{336 l^{3}}\left[(8 l+j)_{l}^{(8)}-(7 l+j)_{l}^{(8)}\right]\right. \\
& \left.\left.\left.-\frac{1}{30 l^{2}}\left[(8 l+j)_{l}^{(7)}\right)-(7 l+j)_{l}^{(5)}\right)\right]-\frac{1}{12 l}\left[(8 l+j)_{l}^{(6)}-(7 l+j)_{l}^{(6)}\right)\right]-\frac{1}{30}\left[(8 l+j)_{l}^{(5)}\right. \\
& \left.\left.\left.-(7 l+j)_{l}^{(5)}\right)\right]\right\}
\end{aligned}
$$

solution: By taking $\mathrm{p}_{1}=2, \mathrm{p}_{2}=3, \mathrm{n}=3, \mathrm{~m}=2$ in corollary 6 , we find $\left[\frac{k}{l}\right]\left[\frac{k}{l}\right]-t\left[\frac{k}{l}\right]-t-s$

$$
\begin{gathered}
\sum_{t=2} \sum_{s=0} \sum_{r=0}(k-t l-s l-r l)^{2}(k-l-t l-s l-r l)^{3}= \\
\frac{k_{l}^{(8)}}{336 l^{3}}+\frac{k_{l}^{(7)}}{30 l^{2}}+\frac{k_{l}^{(6)}}{12 l}+\frac{k_{l}^{(5)}}{30}+c_{2_{j}} \frac{k_{l}^{(2)}}{2 ! l^{2}}+c_{1_{j}} \frac{k}{l}+c_{0_{j}} .
\end{gathered}
$$

putting $k=(7 l+j), k=(8 l+j)$ and $k=(9 l+j)$ in $(20)$, we obtain

$$
\begin{aligned}
& \sum_{t=2}^{7} \sum_{s=1}^{7-t} \sum_{r=0}^{7-t-s}(7 l+j-t l-s l-r l)^{2}(7 l+j-l-t l-s l-r l)^{3}=\frac{(7 l+j)_{l}^{(8)}}{336 l^{3}}+ \\
& \frac{(7 l+j)_{l}^{(7)}}{30 l^{2}}+\frac{(7 l+j)_{l}^{(6)}}{12 l}+\frac{(7 l+j)_{l}^{(5)}}{30}+c_{2_{j}} \frac{(7 l+j)_{l}^{(2)}}{2 ! l^{2}}+c_{1_{j}} \frac{(7 l+j)}{l}+c_{0_{j}} \cdot,(21) \\
& \sum_{t=2}^{8} \sum_{s=1}^{8-t} \sum_{r=0}^{8-t-s}(8 l+j-t l-s l-r l)^{2}(8 l+j-l-t l-s l-r l)^{3}=\frac{(8 l+j)_{l}^{(8)}}{336 l^{3}}+ \\
& \frac{(8 l+j)_{l}^{(7)}}{30 l^{2}}+\frac{(8 l+j)_{l}^{(6)}}{12 l}+\frac{(8 l+j)_{l}^{(5)}}{30}+c_{2_{j}} \frac{(8 l+j)_{l}^{(2)}}{2 ! l^{2}}+c_{1_{j}} \frac{(8 l+j)}{l}+c_{0_{j}} \cdot,(22)
\end{aligned}
$$




$$
\begin{aligned}
& \text { and } \\
& \sum_{t=2}^{9} \sum_{s=1}^{9-t} \sum_{r=0}^{9-t-s}(9 l+j-t l-s l-r l)^{2}(9 l+j-l-t l-s l-r l)^{3}=\frac{(9 l+j)_{l}^{(8)}}{336 l^{3}}+ \\
& \frac{(9 l+j)_{l}^{(7)}}{30 l^{2}}+\frac{(9 l+j)_{l}^{(6)}}{12 l}+\frac{(9 l+j)_{l}^{(5)}}{30}+c_{2_{j}} \frac{(9 l+j)_{l}^{(2)}}{2 ! l^{2}}+c_{1_{j}} \frac{(9 l+j)}{l}+c_{0_{j}} \cdot \text {, (23) } \\
& \text { Solving (21), (22) and (23), we can find } c_{2_{\mathrm{j}}}, \mathrm{c}_{1_{\mathrm{j}}} \text { and } \mathrm{c}_{0_{\mathrm{j}}} \text {. Now, the proof } \\
& \text { follows by substituting the values of } \mathrm{c}_{2_{\mathrm{j}}}, \mathrm{c}_{1_{\mathrm{j}}} \text { and } \mathrm{c}_{0_{\mathrm{j}}} \text { in (20). } \\
& \text { In particular, taking } \mathrm{k}=70 \text { and } l=4 \text {, we get } \\
& \left\{\left[(58)^{2}(54)^{3}+(54)^{2}(50)^{3}+\cdots+(6)^{2}(2)^{3}\right]+\left[(54)^{2}(50)^{3}+(50)^{2}(46)^{3}\right.\right. \\
& \left.\left.+\cdots+(6)^{2}(2)^{3}\right]\right\}+\left\{\left[(54)^{2}(50)^{3}+(50)^{2}(46)^{3}+\cdots+(6)^{2}(2)^{3}\right]+\left[(50)^{2}(46)^{3}\right.\right. \\
& \left.\left.+(46)^{2}(42)^{3}+\cdots+(6)^{2}(2)^{3}\right]\right\}+\cdots+(6)^{2}(2)^{3}=\frac{1}{336(4)^{3}}\left[(70)_{4}^{(8)}-(30)_{4}^{(8)}\right] \\
& \left.+\frac{1}{30(4)^{2}}\left[(70)_{4}^{(7)}\right)-(30)_{4}^{(7)}\right]+\frac{1}{48}\left[(70)_{4}^{(6)}-(30)_{4}^{(6)}\right]+\frac{1}{30}\left[(70)_{4}^{(5)}-(30)_{4}^{(5)}\right] \\
& +\sum_{t=2}^{7} \sum_{s=1}^{7} \sum_{r=0}^{7-s}(7 l+j-t l-s l-r l)^{2}(7 l+j-l-t l-s l-r l)^{3}+(10) \\
& \left\{\sum_{s=1}^{8} \sum_{r=0}^{8-t}(8 l+j-t l-s l)^{2}(8 l+j-l-t l-s l)^{3}-\frac{1}{336(4)^{3}}\left[(34)_{4}^{(8)}-(30)_{4}^{(8)}\right]\right. \\
& \left.\left.-\frac{1}{30(4)^{2}}\left[(34)_{4}^{(7)}\right)-(30)_{4}^{(7)}\right]-\frac{1}{48}\left[(34)_{4}^{(6)}-(30)_{4}^{(6)}\right]-\frac{1}{30}\left[(34)_{4}^{(5)}-(30)_{4}^{(5)}\right]\right\} \\
& \left.+\frac{1}{2 ! l^{2}}\left[(70)_{4}^{(2)}\right)-(30)_{4}^{(2)}+(10)\left[(34)_{4}^{(2)}-(30)_{4}^{(2)}\right]\right] \\
& \left\{\sum_{t=2}^{5}(8 l+j-t l)^{2}(8 l+j-t l-l)^{3}-\frac{1}{336(4)^{3}}\left[(38)_{4}^{(8)}-(30)_{4}^{(8)}\right]\right. \\
& \left.\left.-\frac{1}{30(4)^{2}}\left[(38)_{4}^{(7)}\right)-(30)_{4}^{(7)}\right]-\frac{1}{48}\left[(38)_{4}^{(6)}-(30)_{4}^{(6)}\right]-\frac{1}{30}\left[(38)_{4}^{5}\right)-(30)_{4}^{(5)}\right] \\
& -2\left(-\frac{1}{336(4)^{3}}\left[(34)_{4}^{(8)}-(30)_{4}^{(8)}\right]-\frac{1}{30(4)^{2}}\left[(34)_{4}^{(7)}\right)-(30)_{4}^{(7)}\right] \\
& \left.\left.-\frac{1}{48}\left[(34)_{4}^{(6)}-(30)_{4}^{(6)}\right]-\frac{1}{30}\left[(34)_{4}^{(5)}-(30)_{4}^{(5)}\right]\right)\right\} \\
& =9293238528
\end{aligned}
$$

\section{CONCLUSION}

We conclude that the theory, the results and the applications obtained in this dissertation are derived using the generalized difference operators, their inverses and using the Stirling numbers of the first and second kinds. Theory and applications of the solutions of the generalized equations established in this dissertation, which are not developed earlier are applicable to the area in Numerical Methods.

\section{REFERENCES}

[1] R. P Agarwal. (2000). Difference equations and inequalities. New York: Marcel Dekker.

[2] M. Maria Susai Manuel, G. Britto Antony Xavier, \& E. Thandapani. (2006). Theory of generalized difference operator and its applications. Far East Journal of Mathematical Science, 20(2), 163-171.

[3] M. Maria Susai Manuel, G. Britto Antony Xavier, \& E. Thandapani. (2006). Qualitative properties of solutions of certain class of difference equations. Far East Journal of Mathematical Science, 23(3), 295-304.

[4] M. Maria Susai Manuel, G. Britto Antony Xavier, \& E. Thandapani. (2007). Generalized bernoulli polynomials through weighted pochhammer symbols. Far East Journal of Applied Mathematics, 26(3), 321-333.

[5] M. Maria Susai Manuel, A. George Maria Selvam, \& G. Britto Antony Xavier. (2006). Rotatory and boundedness of solutions of certain class of difference equations, International Journal of Pure and Applied Mathematics, 33(3), 333-343.

[6] M. Maria Susai Manuel \& G. Britto Antony Xavier. (2007). Recessive, dominant and spiral behaviours of solutions of certain class of generalized difference equations. International Journal of Differential Equations and Applications, 10(4), 423-433.

[7] M. Maria Susai Manuel, G. Britto Antony Xavier, \& V. Chandrasekar. (2008). Generalized difference operator of the second kind and its application to number theory. International Journal of Pure and Applied Mathematics, $47(1), 127-140$. 
[8] M. Maria Susai Manuel, G. Britto Antony Xavier, V. Chandrasekar, R. Pugalarasu, \& S. Elizabeth. (2009). On generalized difference operator of third kind and its applications in number theory. International Journal of Pure and Applied Mathematics, 53(1), 69-81.

[9] R. E. Mickens. (1990). Difference equations. New York: Van Nostrand Reinhold Company.
[10] S. N. Elaydi. (1999). An introduction to difference equations. ( $2^{\text {nd }}$ ed.). Springer.

[11] W. G. Kelley \& A. C. Peterson. (1991). Difference equations. An introduction with applications. Academic Press. 